\begin{tabular}{|l|l|l|l|}
\hline Eiszeitalter u. Gegenwart & $\mathbf{2 9}$ & $\begin{array}{c}201-213 \\
7 \mathrm{Abb} ., 1 \mathrm{Tab} .\end{array}$ & Hannover 1979 \\
\hline
\end{tabular}

\title{
Submarine glazial übertiefte Täler vor Südgrönland
}

\author{
Continental shelf, off-shore, bathymetric map, valley, fluviatile erosion (preglacial), glacial erosion \\ Greenland, Greenland Sea \\ Gerd SommerhofF *)
}

\begin{abstract}
Kurzfassung: Auf der Grundlage von Tiefenkarten, Lotprofilen und Sedimentechogrammen werden Topographie und Morphologie südwest- und südostgrönländischer Schelfrinnen erfaßt und in ihrer Genese gedeutet. Danach stellen die Schelfrinnen glazial überformte Täler präglazialer Anlage mit geringer mariner Umgestaltung dar.

Mündungsschwelle, Moränenumrahmung und ein in Becken und Schwellen gegliedertes Längsprofil weisen die Rinnen als glazial übertiefte Tröge und Gletscherbecken mit Übertiefungsbeträgen von durchschnittlich $275 \mathrm{~m}$ aus. Ihre fluviale Anlage fällt in eine Zeit, als die herausgehobenen Kontinentalränder intensiver Abtragung ausgesetzt waren. Die postglaziale marine Sedimentation in den Schelfrinnen ist gering, bedingt durch minimale terrigene Sedimentzufuhr und hohe Fließgeschwindigkeiten des Ost- und Westgrönlandstromes.
\end{abstract}

\section{[Submarine Glacial Troughs off South-Greenland]}

A bstract: Based on detailed bathymetric maps, depth profiles and 3,5-kHz-echograms the physiography and morphology of the shelf valleys off southwest- and southeast-Greenland are analysed. The submarine valleys are ice formed troughs with an average overdeepening of $275 \mathrm{~m}$. The fluvial incision of the shelf valleys took place during a time of uplifted continental margins causing a period of erosion. The postglacial marine sedimentation is thin due to minimal terrigenious sediment supply and high velocities of the East- and Westgreenland currents.

\section{Einleitung}

Die Schelfflächen des südwest- und südostgrönländischen Kontinentalrandes sind durch eine Vielzahl glazial geformter Rinnen tief zerschnitten. Diese starke Zertalung zeigt sich besonders eindrucksvoll in einem küstenparallelen Längsprofil über den SEGrönlandschelf (Abb. 1). In dem Profil sind die Schelfrinnen bis über $600 \mathrm{~m}$ Meerestiefe in die durchschnittlich $200 \mathrm{~m}$ tiefe Schelffläche eingeschnitten. Die Schelffläche ist durch die Schelfrinnen in einzelne Platten gegliedert und insbesondere im nördlichen Profilbereich stark aufgelöst.

Diese submarinen Rinnen werden im folgenden als fluvial erodierte, im Meer ertrunkene und glazial überformte Täler mit geringer mariner Umgestaltung gedeutet. Sie sind durch subaerische, subglaziale und submarine Formungsprozesse geprägt. Hieraus ergibt sich die Gliederung meiner Ausführungen in drei Abschnitte. Der erste Teil befaßt sich mit der glazialen Gestaltung und Übertiefung der Schelfrinnen; im zweiten Teil wird ihre präglaziale fluviale Anlage diskutiert, während im dritten und letzten Abschnitt die spät- und postglaziale marine Überformung skizziert wird.

\section{Glaziale Gestaltung und Übertiefung der Schelfrinnen}

Die Schelfgebiete vor SW- und SE-Grönland gehören zu den glazial geprägten Schelfen (Rvachev 1964; Holtedahl 1970; Henderson 1975; Sommerhoff 1975 a, b). Neben den küstennahen Rundhöckerflächen des Strandflateschelfs und den Eisrandablagerun-

*) Anschrift des Verfassers: Dr. G. S o m m e r h of f, Institut für Geographie der Universität München, Luisenstraße 37, 8000 München 2. 

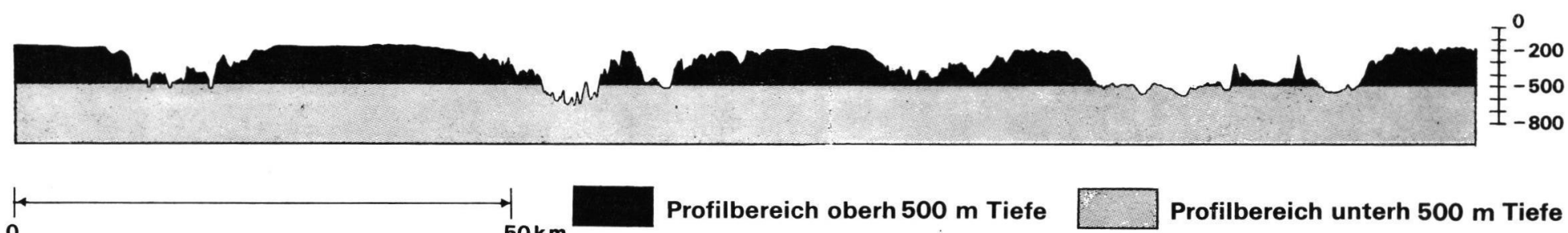

Profilbereich oberh 500 m Tiefe

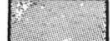

Profilbereich unterh $\mathbf{5 0 0} \mathrm{m}$ Tiefe

Abb. 1: Küstenparalleles Profil über die Schelfrinnen des SE-Grönlandschelfs (Grundlage: Echogramme von FS. „Meteor“-Fahrt 6/1966. Lage in Abb. 2). 


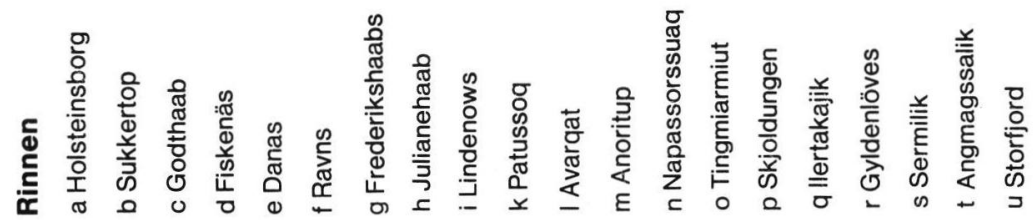

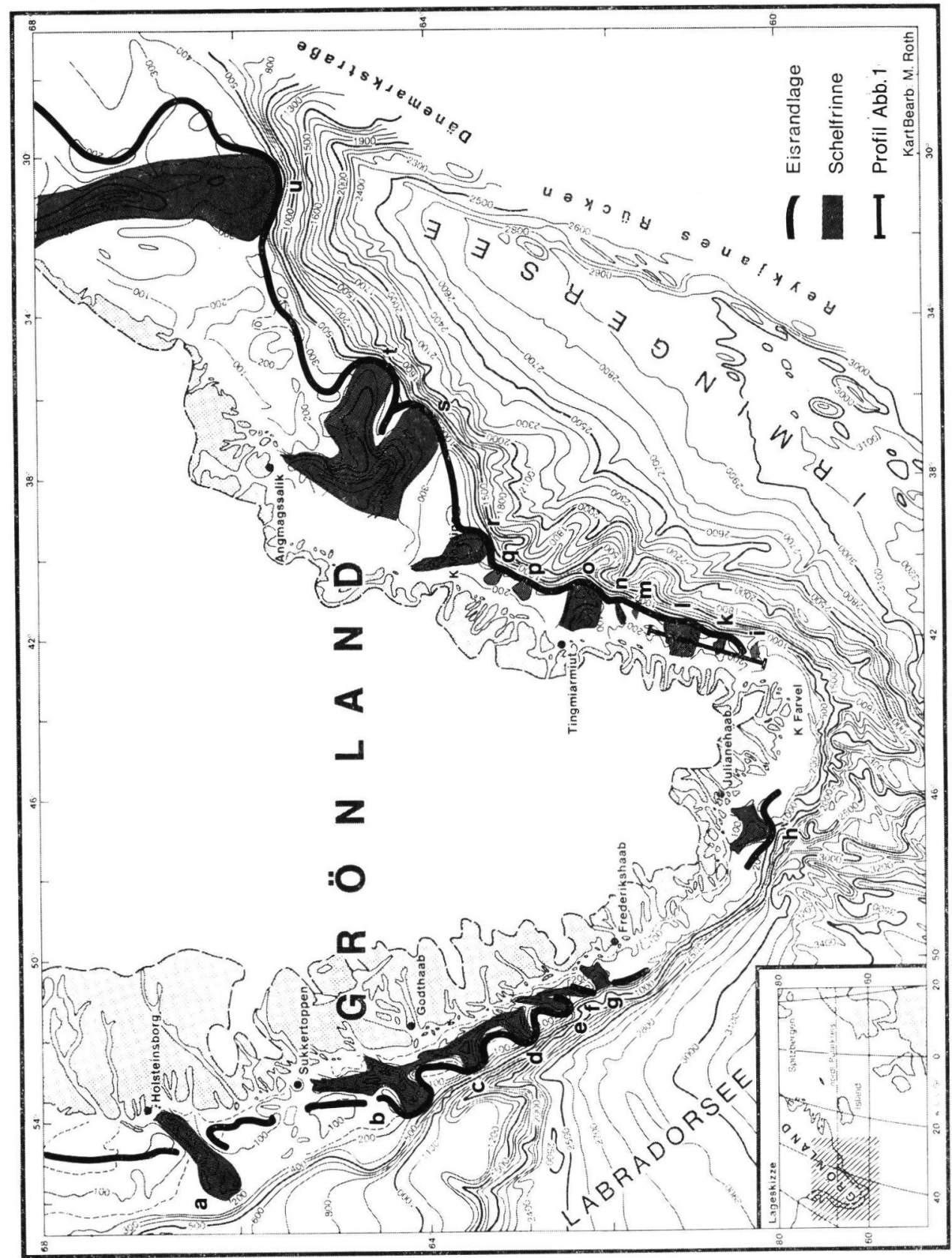

Abb. 2: Tiefenkarte des südgrönländischen Kontinentalrandes mit Schelfrinnen (Grundlage: Seeund Vermessungskarten des Dänischen, Deutschen und Kanadischen Hydrographischen Dienstes). Merkator-Projektion. 
gen der Schelfbänke gehören die Schelfrinnen zu den auffallendsten Elementen des submarinen glazialen Formenschatzes.

Topographie und Bathymetrie der Schelfrinnen sind in Abb. 2 in einer Tiefenkarte des grönländischen Kontinentalrandes dargestellt. Danach läßt sich das Rinnensystem des Schelfs in küstenparallele Längs- und senkrecht bis quer dazu verlaufende Querrinnen gliedern. Während vor SW-Grönland die Längsrinnen eine zusammenhängende, nur durch Schwellen gegliederte marginale Tiefenzone bilden, ist auf dem SE-Grönlandschelf ein System langhinziehender Längsrinnen nicht entwickelt. Nur bei der Gyldenlöves- und Angmagssalik-Sermilik-Rinne ist eine deutliche Längsrinnen-Entwicklung festzustellen.

Die Querrinnen des SE-Grönlandschelfs liegen in unmittelbarer Verlängerung der Fjorde und streichen in gleicher Richtung. Sie sind daher als deren untermeerische Fortsetzung anzusehen. Die großen Querrinnen des SW-Grönlandschelfs stellen dagegen keine Verlängerungen der Fjorde dar. Sie nehmen vielmehr ihren Anfang auf dem Schelf, wo sie aus den Längsrinnen hervorgehen. Die Fjorde setzen sich nur auf dem Küstenschelf in steilen, trogförmigen Einschnitten fort. Diese Küstenschelf-Rinnen münden in die Sammeladern der Längsrinnen.

Feinrelief und Morphologie der Schelfrinnen sollen exemplarisch an einer bathymetrischmorphologischen Detailkarte der Sukkertop-Rinne verdeutlicht werden (Abb. 3). Die Tiefenkarte mit einer Äquidistanz von $20 \mathrm{~m}$ und $10 \mathrm{~m}$ Zwischenlinien im Bereich der Schelfbänke basiert auf dänischen Original-Vermessungskarten. Grundlage für die morphologische Interpretation bildet eine Feinreliefanalyse der engabständigen $(1-2 \mathrm{~km})$ dänischen Lotprofile.

Die Sukkertop-Rinne setzt sich aus einem Längs- und Querrinnen-Abschnitt zusammen. Die Längsrinne bildet die Grenze zwischen dem unruhigen Rundhöckerrelief des küstenwärtigen Strandflate-Schelfs und den glazialen Akkumulationsformen des Außenschelfs, während durch die Querrinne zwei Schelfbänke, die Fyllas- und die Banan-Bank, voneinander getrennt werden. Der Rundhöckerschelf in 100-200 m Tiefe ist durch steile Trogtäler tief zerschnitten.

Längs- und Querrinne werden auf dem Außenschelf von einem langgestreckten, rinnenparallelen Moränenrücken der Schelfbänke flankiert. Dieser Rücken, der die Kammlinie der Schelfbänke bildet und unmittelbar an die Sukkertop-Rinne grenzt, ist als submarine Eisrandlage zu deuten (Sommerhoff 1975 a, b). Am Schelfrand geht er in die flache Mündungsschwelle der Sukkertop-Rinne über, wodurch diese meerwärts halbkreisförmig abgeriegelt wird und mit deutlichem Gegengefälle am Kontinentalhang mündet. Der Verlauf der Eisrandlage zeigt, daß das pleistozäne Schelfeis in den Schelfrinnen am weitesten meerwärts vorgestoßen ist. In den Querrinnen haben somit die Auslaßgletscher einer bis zu den Längsrinnen reichenden zusammenhängenden Schelfvereisung gelegen.

Die Mikrobodentopographie der Mündungsschwelle soll an einem Echogramm-Bcispiel verdeutlicht werden (Abb. 4). In dem Profil steigt der Rinnenboden von $300 \mathrm{~m}$ Tiefe mit sanftem Gegengefälle von $4 \%$ bis auf $253 \mathrm{~m}$ am Schelfrand an, wo er mit markantem Gefällsknick in den steilen Kontinentalhang übergeht. Die Entstehung dieser Mündungsschwellen der Schelfrinnen ist auf zwei Ursachen zurückzuführen: Einmal ist sie bedingt durch ein Nachlassen der Gletschererosion am Schelfrand, verursacht durch das Aufschwimmen des Schelfeises über dem Kontinentalhang, zum anderen durch submarine Eisrandablagerungen. Serien niedriger $(2-5 \mathrm{~m})$, dicht gestaffelter Wälle können als Kalbungsmoränen gedeutet werden. Sie entstehen, wenn beim Kalbungsvorgang die schwimmende Gletscherzunge in Schwingungen gerät und durch Bodenberührung die Sedimente wallartig zusammenstaucht. Auch ein im Rhythmus der Gezeiten alternierendes Aufschwimmen und Auflasten des Eises kann zu einem Ausquetschen von Sedimenten an der Kalbungsfront führen. 

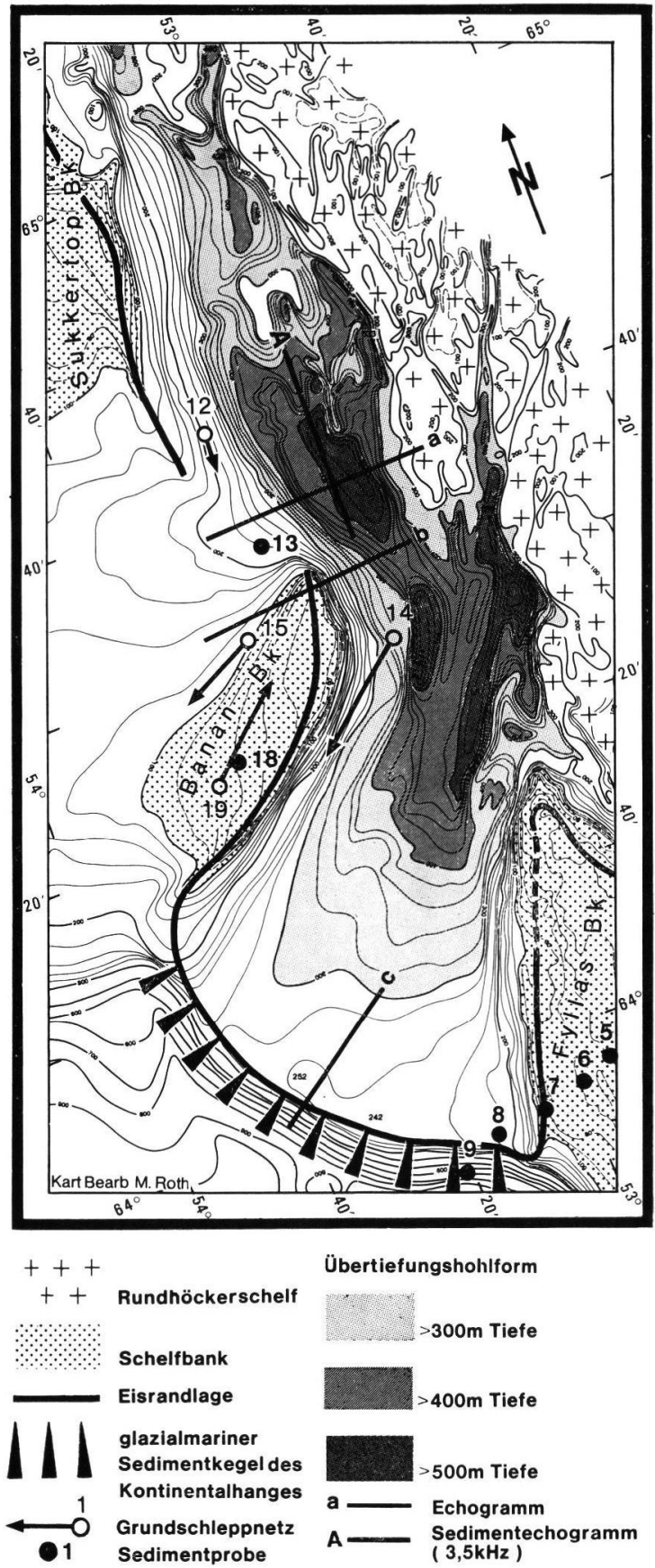

Abb. 3: Bathymetrisch-morphologische Detailkarte der Sukkertop Rinne (Grundlage: OriginalVermessungskarten des „Kongelige Danske Sökort Arkiv, Kopenhagen). Merkatorprojektion. 


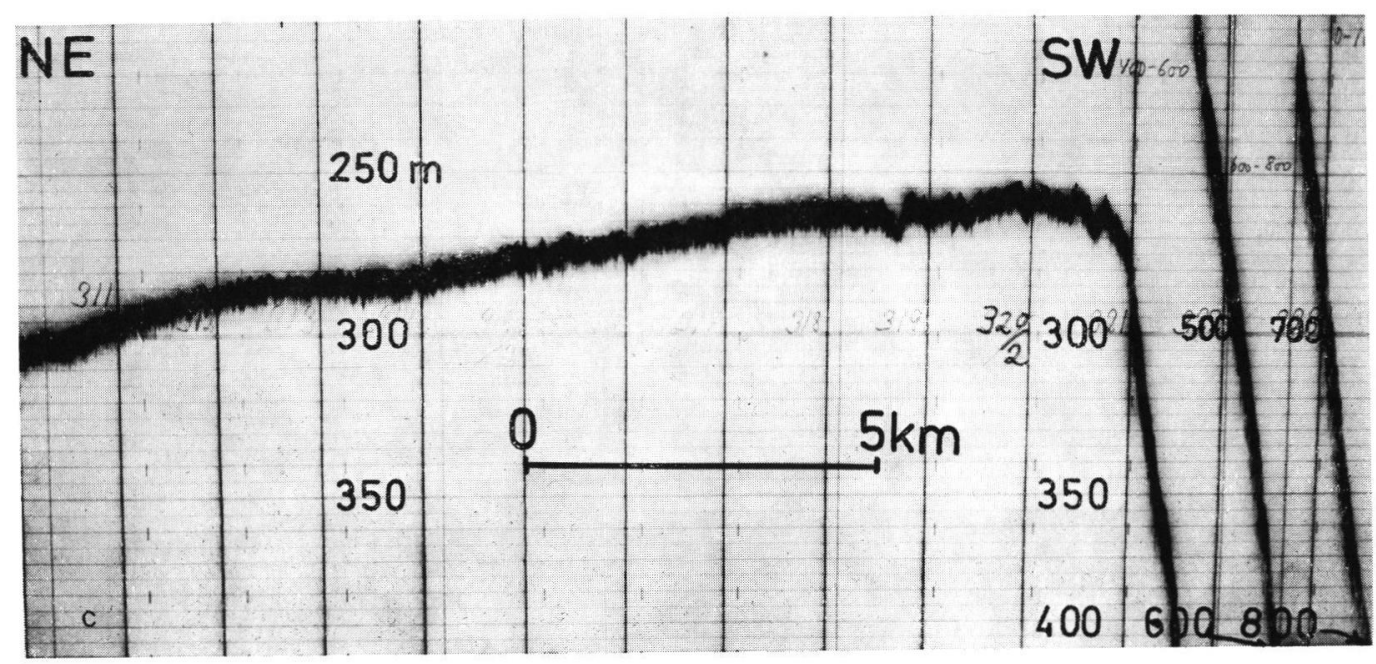

Abb. 4: Echogramm der Mündungsschwelle der Sukkertop-Rinne (aufgenommen vom „Kongelige Danske Sökort Arkiv, Kopenhagen. Lage in Abb. 3: Profil c).

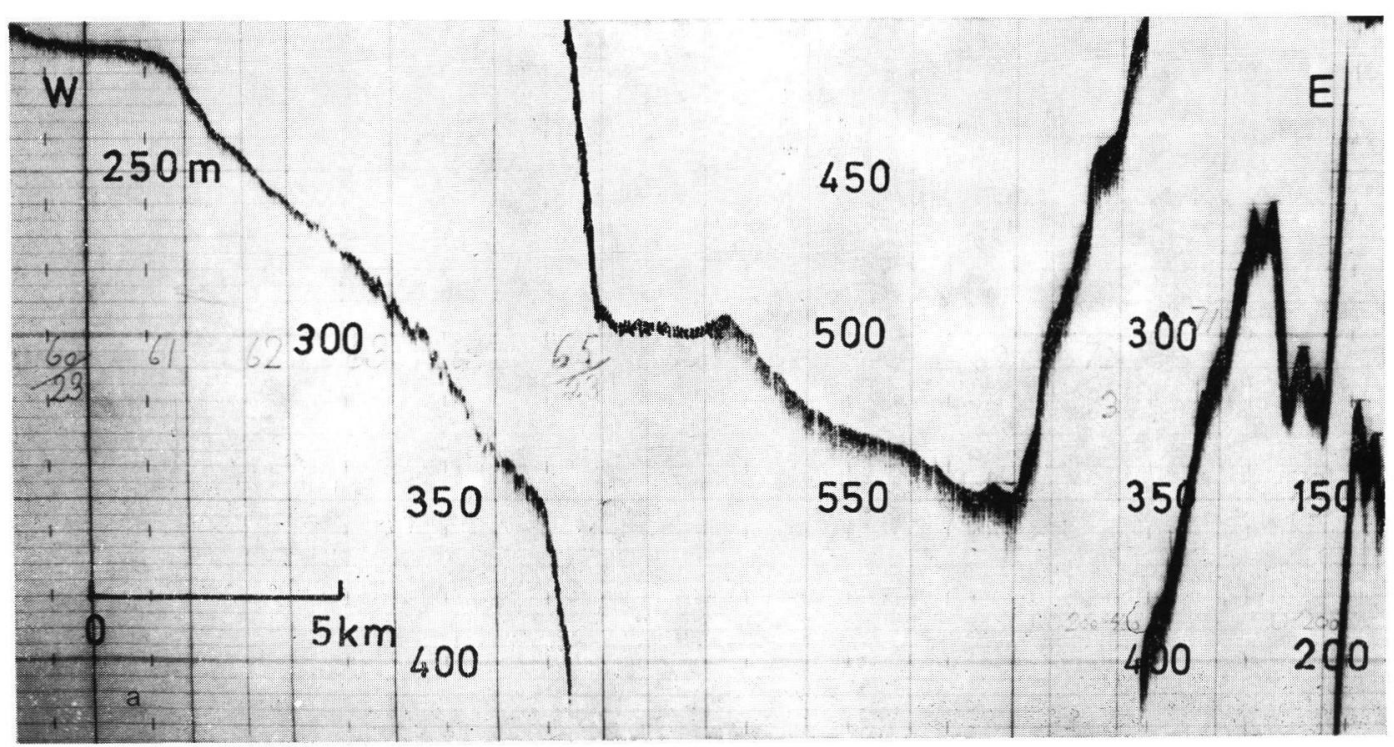

Abb. 5: Echogramm quer zur Sukkertop-Längsrinne (aufgenommen vom „Kongelige Danske Sökort Arkiv, Kopenhagen. Lage in Abb. 3: Profil a). 
Die Längsrinnen sind durch eine auffallende Talasymmetrie mit steilerem E- und flacherem W-Hang gekennzeichnet. Die bereits in der Tiefenkarte (Abb. 2) zu erkennende Asymmetrie wird an dem Profil a in Abb. 5 illustriert. In dem Echogramm fällt die westliche Flanke der Sukkertop-Rinne mit durchschnittlich $2^{\circ}$ von 215 auf $350 \mathrm{~m}$ Tiefe und geht hier in eine $150 \mathrm{~m}$ hohe Steilstufe von $8-9^{\circ}$ über, an die sich in $500 \mathrm{~m}$ Wassertiefe eine $1,9 \mathrm{~km}$ breite Hangverflachung anschließt, die $\mathrm{zu}$ einem unteren $50 \mathrm{~m}$ hohen Flachhang weiterleitet. Der E-Hang fällt mit einer $200 \mathrm{~m}$ hohen und $20^{\circ}$ steilen Stufe von der küstenwärtigen Rundhöckerfläche in $120 \mathrm{~m}$ auf $320 \mathrm{~m}$ Tiefe ab. Hier wird der weitere Abfall durch eine $60 \mathrm{~m}$ hohe Aufragung unterbrochen und setzt sich danach mit $5^{\circ}$ Neigung bis zur Rinnensohle in $550 \mathrm{~m}$ Tiefe fort. Die größte Tiefe dieses Profils liegt charakteristischerweise am Fuß des östlichen Steilhanges.

Die Talasymmetrie weist auf unterschiedliche petrographische Resistenz der Talhänge hin und spricht für Holtedahls (1970) Deutung der Längsrinnen als geologisch-tektonische Grenzlinie zwischen den präkambrischen Gneisen des Küstenschelfs und weniger resistenten jüngeren (mesozoischen) Sedimentgesteinen des Außenschelfs.

Die Sukkertop-Rinne ist durch breite und flache Schwellen in mehrere übertiefte Teilbecken gegliedert (Abb. 3). Einige dieser Becken sind über $500 \mathrm{~m}$ tief. Als maximale Tiefe wurden $635 \mathrm{~m}$ am Zusammenfluß von Längs- und Querrinne unmittelbar am Fuß des Steilhangabfalles des Rundhöckerschelfs gemessen. Diese Erscheinung kann ebenso bei der Fiskenäs-, Danas-, Ravns- und anderen Rinnen beobachtet werden und ist als glaziale Übertiefung durch Konfluenz pleistozäner Gletscher zu deuten, worauf bereits HoLTEDAHL (1970) hingewiesen hat.

Mündungsschwelle, Moränenumrahmung und ein in Becken und Schwellen gegliedertes Längsprofil weisen die Sukkertop-Rinne als glazial übertieftes Gletscherbecken aus.

Schelfrand und Kontinentalhang sind vor den Schelfrinnen nicht als canyonförmige Einschnitte ausgebildet, in denen sich die Schelfrinnen meerwärts fortsetzen, sondern sind vielmehr durch deltaförmige Sedimentkegel meerwärts vorgebaut (Abb. 2 u. 3). Dieser sedimentäre Schelfvorbau beträgt bei der Sukkertop-Rinne ca. $7 \mathrm{~km}$. Ahnliche Sedimentkegel sind vor fast allen Rinnen ausgebildet. Eine Ausnahme machen nur die Ravns- und Frederikshaabs-Rinne. Der Ansatz der Sedimentkegel an den Eisrandablagerungen weist sie als glazimarine Ablagerungen aus. Ihre Mächtigkeit deutet jedoch auf eine längere Ablagerungszeit und damit auf eine bereits präpleistozäne Anlage hin (vgl. Kap. 2).

Über die Eintiefungs- und Übertiefungsbeträge der Schelfrinnen gibt Tab. 1 Auskunft. Danach sind die Rinnen zwischen $210 \mathrm{~m}$ (Napassorssuaq-Rinne) und $845 \mathrm{~m}$ (GyldenlövesRinne) in die Schelffläche eingearbeitet. Als Mittelwert wurde für den SE-Grönlandschelf ein maximaler Eintiefungsbetrag von $430 \mathrm{~m}$ und für den SW-Grönlandschelf von $470 \mathrm{~m}$ errechnet. Die Mündungsschwellen sind zwischen $195 \mathrm{~m}$ (Holsteinsborg-Rinne) und $470 \mathrm{~m}$ (Avarqat-Rinne) tief. Vor SE-Grönland liegen sie im Mittel $65 \mathrm{~m}$ tiefer als vor SWGrönland, wo sie eine durchschnittliche Tiefe von $300 \mathrm{~m}$ erreichen.

Die Übertiefung kann nur größenordnungsmäßig erfaßt werden. Ein brauchbarer Ansatz scheint mir die Differenz zwischen maximaler Rinnentiefe und maximaler Schwellentiefe zu sein. Hierbei handelt es sich um einen maximalen Wert, bei dem vorausgesetzt wird, daß die Mündungsschwelle im präglazialen Untergrund ausgebildet ist und nicht durch Sedimente, insbesondere durch Eisrandablagerungen, stark überhöht ist. Dieser Ansatz kommt aber der Realität sehr nahe, da ja auch bei den maximalen Rinnentiefen eventuelle eiszeitliche und nacheiszeitliche Ablagerungen ebenso unberücksichtigt bleiben und sich im Mittel beide Werte größenordnungsmäßig aufheben. Im Mittel beläuft sich die Übertiefung auf $275 \mathrm{~m}$. Die Werte der maximalen Übertiefungen streuen zwischen $115 \mathrm{~m}$ (Napassorssuaq-Rinne) und $690 \mathrm{~m}$ (Gyldenlöves-Rinne). Obwohl die Schelfrinnen 
Tab. 1: Eintiefungs- und Übertiefungsbeträge südgrönländischer Schelfrinnen (Maxima in $\mathrm{m} \cdot$ Lage der Rinnen in Abb. 2)

\begin{tabular}{|c|c|c|c|c|c|}
\hline \multirow{3}{*}{ 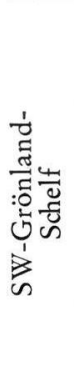 } & Schelfrinnen & Rinnentiefe & Schwellentiefe & Eintiefung1) & Ubertiefung 2 ) \\
\hline & $\begin{array}{l}\text { Holsteinsborg } \\
\text { Sukkertop } \\
\text { Godthaab } \\
\text { Fiskenäs } \\
\text { Danas } \\
\text { Ravns } \\
\text { Frederikshaabs } \\
\text { Julianehaab }\end{array}$ & $\begin{array}{l}545 \\
635 \\
605 \\
505 \\
\mathbf{4 2 0} \\
555 \\
515 \\
600\end{array}$ & $\begin{array}{l}195 \\
280 \\
295 \\
280 \\
210 \\
415 \\
360 \\
350\end{array}$ & $\begin{array}{l}480 \\
575 \\
545 \\
435 \\
350 \\
480 \\
435 \\
450\end{array}$ & $\begin{array}{l}350 \\
355 \\
310 \\
225 \\
210 \\
140 \\
155 \\
250\end{array}$ \\
\hline & Mittel & 550 & 300 & 470 & 250 \\
\hline \multirow[t]{3}{*}{ 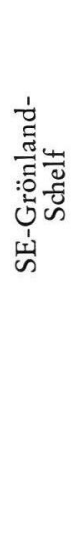 } & $\begin{array}{l}\text { Lindenows } \\
\text { Patussoq } \\
\text { Avarqat } \\
\text { Anoritup } \\
\text { Napassorssuaq } \\
\text { Tingmiarmiut } \\
\text { Skjoldungen } \\
\text { Ilertakajik } \\
\text { Gyldenlöves } \\
\text { Sermilik } \\
\text { Angmagssalik } \\
\text { Storfjord }\end{array}$ & $\begin{array}{r}570 \\
550 \\
700 \\
530 \\
\mathbf{4 2 0} \\
700 \\
610 \\
445 \\
\mathbf{1 0 6 0} \\
\mathbf{8 0} \\
850 \\
620\end{array}$ & $\begin{array}{l}330 \\
350 \\
\mathbf{4 7 0} \\
335 \\
305 \\
395 \\
370 \\
325 \\
370 \\
375 \\
375 \\
395\end{array}$ & $\begin{array}{l}400 \\
380 \\
510 \\
250 \\
210 \\
480 \\
380 \\
230 \\
845 \\
650 \\
420\end{array}$ & $\begin{array}{l}240 \\
200 \\
230 \\
195 \\
115 \\
305 \\
240 \\
120 \\
690 \\
475 \\
475 \\
225\end{array}$ \\
\hline & Mittel & 640 & 365 & 430 & 290 \\
\hline & Mittel gesamt & 600 & 340 & 450 & 275 \\
\hline
\end{tabular}

1) Eintiefung $=$ Differenz zwischen maximaler Rinnentiefe und mittlerer Banktiefe.

2) Ubertiefung $=$ Differenz zwischen maximaler Rinnentiefe und maximaler Schwellentiefe.

während der Eiszeiten als Hauptabflußbahnen des grönländischen Inlandeises gedient haben, können diese großen Übertiefungsbeträge nicht allein durch Glazialerosion erklärt werden. Denn bei maximalen Rinnentiefen von durchschnittlich $600 \mathrm{~m}$ (Tab. 1), ist davon auszugehen, daß das Eis über lange Zeiten der Vereisungsphase des Schelfs nicht dem Rinnenboden aufgelegen, sondern vielmehr aufgeschwommen ist. Welche Rolle subglaziale, unter hydrostatischem Druck stehende Strömungen oder der von TiETzE (1961) geforderte, durch den Tidenhub einer schwimmenden Gletscherzunge bedingte Siphoneffekt gespielt haben, läßt sich erst nach Untersuchungen an rezenten Eisschelfen entscheiden. Ferner ist zu prüfen, inwieweit tektonische Vorgänge mitverantwortlich sind für die hohen Übertiefungsbeträge in den geologisch vorgezeichneten Längsrinnen. Möglicherweise wurde diese submarine Störungszone durch glazialisostatische Bewegungen wieder reaktiviert. 


\section{Präglaziale Anlage der Schelfrinnen}

Die Schelfrinnen tragen zwar deutliche Spuren glazialer Gestaltung und Übertiefung, können aber in ihrer ursprünglichen Anlage nicht vom Schelfeis geschaffen sein. Wie für die Fjorde, so ist auch für die Schelfrinnen eine präglaziale Talanlage erforderlich, die vom Eis zu Trogtälern und Gletscherbecken umgestaltet wurde. Für die fluviale Zerschneidung der Schelffläche muß eine um 300-400 m höhere Lage der Schelffläche vorausgesetzt werden. Daß es solche Erosionsperioden, bedingt durch eine höhere Lage des Schelfs, ge-

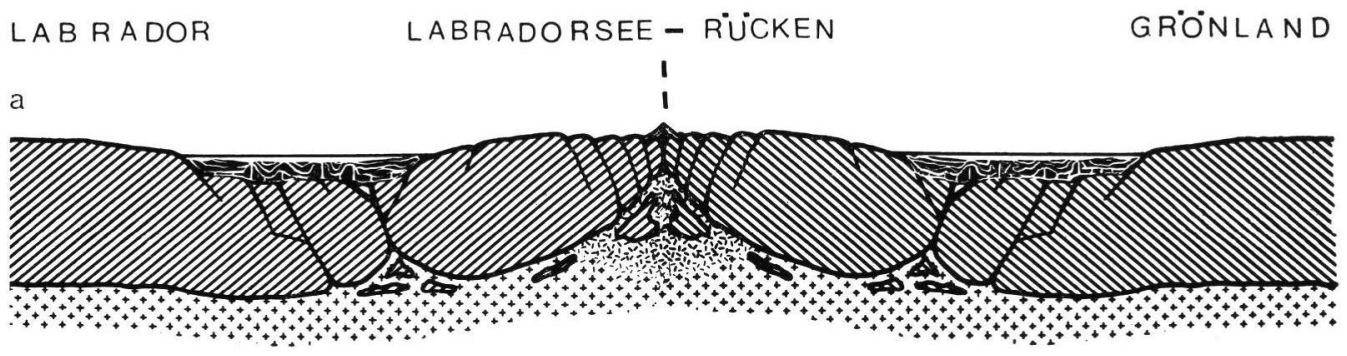

b

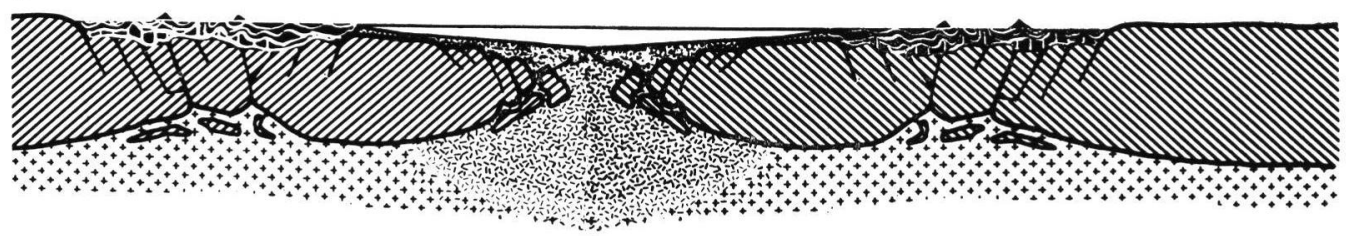

C

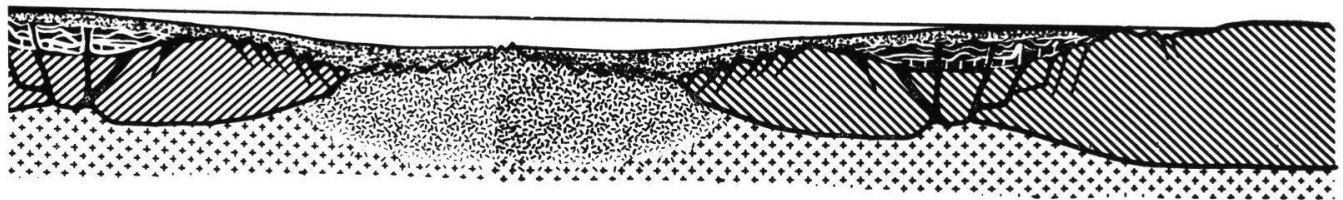

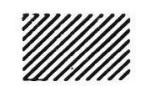

Kontinentale Kruste

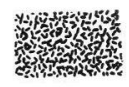

Ozeanische Kruste

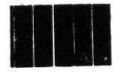

Evaporite

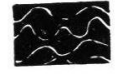

Mesozoisch-paläozoische (?) Sedimente

Mesozoisch-känozoische Sedimente

Abb. 6: Geologische Entwicklung des südwestgrönländischen Kontinentalrandes. Schematische Darstellung der plattentektonischen Deutung nach VAN DER LiNDEN 1975. - a: Paläozoisch-mesozoische Aufwölbung der kontinentalen Kruste im Bereich der späteren Labradorsee und Bildung marginaler Flachmeerbecken. - b: Kreidezeitliche Offfnung der Labradorsee und Hebung der Kontinentalränder. - c: Tertiäre Absenkung der Kontinentalränder. - Phase b und c stellen für die Kontinentalränder der Labradorsee Erosionsperioden mit fluvialer Zertalung der Schelfflächen dar. 
geben hat, zeigt neben morphologischen Befunden die geologische Entwicklung der Labradorsee und ihrer Kontinentalränder. Sie soll daher in Anlehnung an VAN DER LINDEN (1975) und VAN DER LINDEN et al. (1976) kurz skizziert werden.

Nach der plattentektonischen Deutung der Labradorsee wird die kontinentale Kruste im Bereich der späteren Labradorsee während des Paläozoikums emporgehoben. Durch korrespondierende Absenkung der marginalen Bereiche kommt es zur Bildung randlicher Flachmeere mit entsprechenden Ablagerungen von Evaporiten und mesozoisch-paläozoischen (?) Flachmeersedimenten (Abb. 6: Phase a).

Während der Kreide findet eine Reliefumkehr statt: Das zentrale Labradorsee-Becken öffnet sich an dem heute inaktiven Labradorsee-Rücken, während die Kontinentalränder emporgehoben werden (Abb.6: Phase b). Für sie setzt hiermit eine Erosionsperiode ein, während der auf dem Labradorschelf mehrere $100 \mathrm{~m}$ jurassischer Sedimente abgetragen wurden. Im Eozän hört die Ausdehnung der Labradorsee auf und die Kontinentalränder sinken langsam zu ihrem heutigen Niveau ab, womit eine neue Sedimentationsphase eingeleitet wird (Abb. 6: Phase c). Phase b und c stellen für die Kontinentalränder der Labradorsee Erosionsperioden dar, in die die fluviale Anlage der Schelfrinnen fällt.

Für die plattentektonische Deutung der geologischen Entwicklung der Labradorsee und ihrer Kontinentalränder sprechen eine Vielzahl geologisch-geophysikalischer Indizien. Die Entdeckung des unter Sedimenten begrabenen Labradorsee-Rückens durch DraKe et al. (1963) war ein erster Hinweis auf die Entstehung der Labradorsee durch Prozesse des „sea floor spreading“. Dieses Indiz wurde bestätigt durch die Ergebnisse magnetischer Untersuchungen, wonach sich magnetische Anomalien in einem parallelen Streifenmuster zum Labradorsee-Rücken anordnen (SRIVASTAVA 1977).

Für eine ehemals höhere Lage der Kontinentalränder und tertiäre Absinkvorgänge sprechen neben der plattentektonischen Deutung der Labradorsee Erdölbohrungen und morphologische Befunde. So wurden nach der Bohrung Leif E 38 (TEnneco 1973) auf dem südlichen Labradorschelf $300-400 \mathrm{~m}$ Deltasedimente gefunden, die der in das Hamilton Inlet mündende Churchill River an der Wende Pliozän-Pleistozän auf der Hamilton Bank abgelagert hat. Das weist auf einen im Spättertiär kontinuierlich absinkenden Schelf hin. Am Kontinentalhang des nördlichen Labradorschelfs (Saglek-Bank) wurden von Fillon et al. (1977) Terrassen zwischen 130 und $400 \mathrm{~m}$ registriert. Die tieferen dieser Terrassen können durch glazial-eustatische Meeresspiegelabsenkung allein nicht erklärt werden. Auf dem SW-Grönlandschelf gibt sich westlich der Sukkertop-Längsrinne eine breite Terrassenfläche zu erkennen, die sich vom Schelfrand in $290 \mathrm{~m}$ küstenwärts bis zu einer Stufe in $260 \mathrm{~m}$ erstreckt (Abb. 2). Ihre Tiefenlage deckt sich mit der maximalen Schwellentiefe der südlich angrenzenden Sukkertop-Querrinne von $280 \mathrm{~m}$ und kommt dem Mittelwert der maximalen Schwellentiefen von $300 \mathrm{~m}$ für die südwestgrönländischen Rinnen sehr nahe (Tab. 1). Daraus darf auf eine ehemalige Erosionsbasis in diesem Niveau geschlossen werden. Auf ein ähnliches Meeresspiegelniveau weisen auch die mächtigen Sedimentkegel vor den Schelfrinnen hin, die als Deltaablagerungen ehemaliger Schelfflüsse zu deuten sind.

Diese morphologischen Befunde sprechen damit ebenfalls für eine ehemals höhere Lage des südwestgrönländischen Kontinentalrandes und weisen auf die präglaziale fluviale Anlage der Schelfrinnen hin.

\section{Spät- und postglaziale marine Überformung der Schelfrinnen}

Von Bedeutung für die spät- und postglaziale marine Umgestaltung der Schelfrinnen und der sie flankierenden Schelfbänke sind eustatische Meeresspiegelschwankungen, Meeresströmungen und terrigene Sedimentzufuhr. Diese Prozesse bestimmen Art und Ausmaß mariner Erosion und Sedimentation auf dem südgrönländischen Kontinentalrand. 


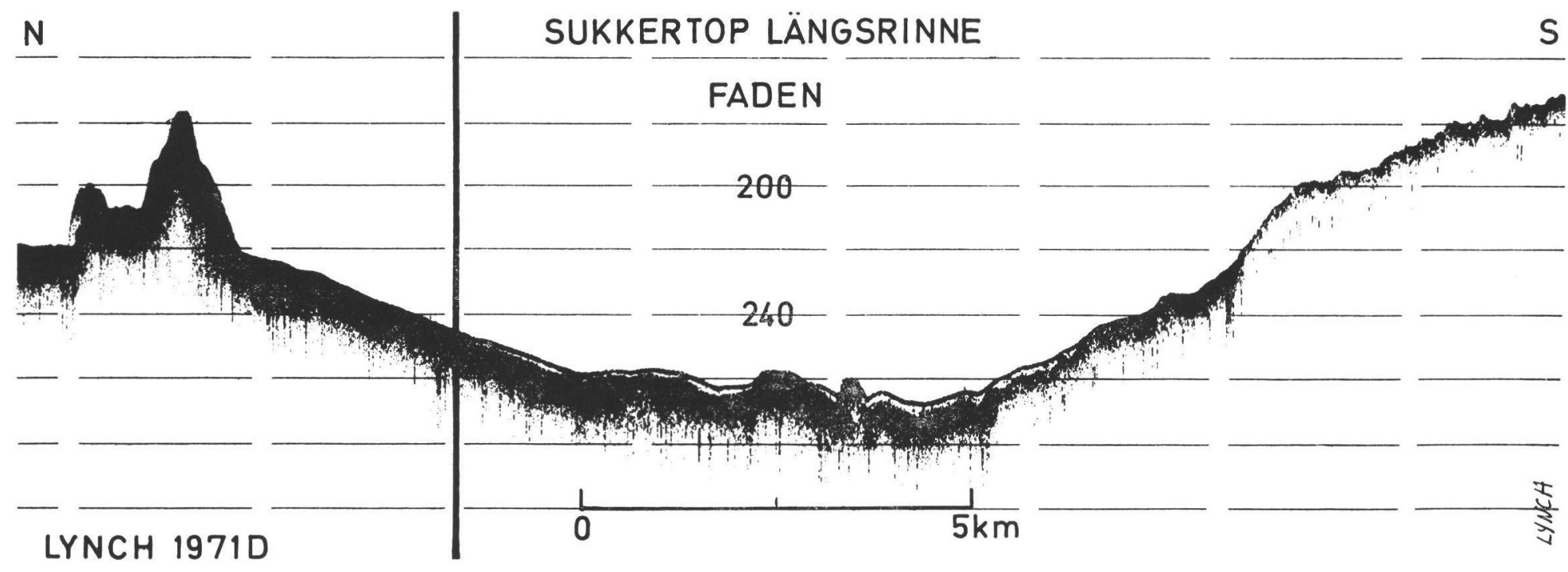

Abb. 7: Sedimentechogramm (3,5 kHz-Echogramm) der Sukkertop-Längsrinne (aufgenommen vom US Navy Department mit USNS „Lynch“. Lage in Abb. 3: Profil A). 
Eine Vorstellung von der postglazialen marinen Sedimentation vermittelt ein Sedimentechogramm (3,5 kHz-Echogramm) der Sukkertop-Längsrinne. Das in Abb. 7 wiedergegebene Profil wurde von dem US-Navy-Forschungsschiff "Lynch" aufgenommen und mir während eines Forschungsaufenthaltes am Navy Department: Naval Ocean Research and Development Activity in Bay St. Louis, Mississippi zur Verfügung gestellt. Hierfür gilt mein besonderer Dank J. Egloff, T. Holcombe und G. L. Johnson. Das Profil verläuft in N-S-Richtung über eines der tiefsten Becken der Sukkertop-Rinne (Lage in Abb. 3). Die maximale Profiltiefe liegt bei $500 \mathrm{~m}$. Deutlich gibt sich eine akustisch transparente Schicht über einem akustisch stark reflektierenden Untergrund zu erkennen. Weniger deutlich ausgeprägt ist ein zweiter, nicht kontinuierlicher interner Reflektor dicht über dem schallharten Untergrund. Die akustisch durchlässige Oberschicht mit interner Schichtung keilt am nördlichen und südlichen Beckenhang in ca. $440 \mathrm{~m}$ Tiefe aus. Der schallharte Untergrund ist stark reliefiert und weist am südlichen Rinnenhang das gleiche kleinkuppige Moränenrelief wie die obere unverschüttete Hangpartie auf. Daraus darf geschlossen werden, daß es sich hier um den glazialen Untergrund handelt, der im Beckentiefsten von postglazialen Sedimenten bedeckt ist.

Die Mächtigkeit der nacheiszeitlichen Sedimentdecke liegt bei durchschnittlich $3 \mathrm{~m}$; die maximale Sedimentmächtigkeit beträgt 5-6 m. Diese Angaben gehen von einer Schallgeschwindigkeit von $1500 \mathrm{~m} \mathrm{sec}^{-1}$ aus, wie sie für die stark wasserhaltigen oberen Sedimentschichten üblich ist. Die postglaziale Sedimentation beschränkt sich auf den unteren, über $400 \mathrm{~m}$ tiefen Beckenboden, während die obere Beckenumrahmung frei von akustisch faßbarer mariner Sedimentation ist. Das zeigt, daß die marine Sedimentation in den Schelfrinnen als gering zu veranschlagen ist.

Hierfür sind mehrere Ursachen maßgebend: Einmal wirken die Fjorde als Sedimentfallen, in denen der größte Teil c'es terrigen zugeführten Materials zum Absatz kommt; zum anderen gelangt das über den Küstenbereich meerwärts verfrachtete Feinmaterial in den Bereich starker Oberflächen- und Tiefenströmungen des Ost- und Westgrönlandstromes, von denen es weiter transportiert wird. Zu einer Ablagerung kommt es nur, wo sich die Strömungsgeschwindigkeiten verringern, wie z. B. an der Südspitze Grönlands. Hierauf sind die mächtigen Akkumulationen des sogenannten „Erich“-Sedimentrückens zurückzuführen, der südlich Kap Farvel am Kontinentalhang als breiter SW-streichender Rücken ansetzt (Abb. 2). Im allgemeinen liegen aber die Strömungsgeschwindigkeiten auf dem südlichen Grönlandschelf über $4 \mathrm{~cm} \mathrm{sec}^{-1}$. Am Schelfrand werden im Bereich der ozeanischen Polarfront, der Konvergenzzone arktischer und atlantischer Wassermassen, Geschwindigkeiten von $25 \mathrm{~m} \mathrm{sec}-$ erreicht (Dietrich, 1957). Strömungsgeschwindigkeiten von $4 \mathrm{~cm} \mathrm{sec}^{-1}$ reichen nach dem bekannten Diagramm von HJuLström (1935) für den Weitertransport von Korngrößen bis $0,5 \mathrm{~mm}$ (Mittelsand) aus. Das bedeutet, daß der Großteil des rezent zugeführten terrigenen Materials nicht zum Absatz kommt, sondern weiter transportiert wird.

Durch die glazialeustatische Meeresspiegelabsenkung von ca. $120 \mathrm{~m}$ (Milliman \& EMERY, 1968), sind weite Gebiete der über $100 \mathrm{~m}$ aufragenden Schelfbänke vor SW-Grönland trockengefallen. Die Transgression des Meeresspiegelanstiegs zeigt sich in Abrasionsterrassen, die durch kleine Kliffs voneinander getrennt werden, in Brandungswällen und -rinnen sowie in einer guten Korngrößensortierung der Banksedimente (Sommerhoff, 1975 a). Das von den Bänken weggespülte Feinmaterial hat jedoch nicht zu einer verstärkten Sedimentation in den Schelfrinnen geführt. Hierfür konnten weder in den Echogrammen noch in den auf einer Forschungsfahrt mit FFS. „Walther Herwig“ entnommenen Sedimentproben Hinweise gefunden werden. Die Ursache ist in einer Verstärkung der Meeres- und Gezeitenströmungen in den Schelfrinnen zu sehen, bedingt durch den Düseneffekt der durch die Bank-Inseln eingerahmten Schelfrinnen. 
Die Untersuchungen zur Morphologie submariner glazial übertiefter Täler beruhen auf marinen „remote sensing“-Verfahren. Eine Überprüfung durch marine "ground checks" wurde durch die Entnahme von Sedimenten auf einer Forschungsfahrt mit FFS. „Walther Herwig “ Ende 1976 durchgeführt. Die erst zum Teil abgeschlossenen sedimentologischen Untersuchungen, über die demnächst an anderer Stelle berichtet wird, bestätigen die hier dargelegten Ergebnisse.

\section{Schriftenverzeichnis}

Dietrich, G. (1957): Schichtung und Zirkulation der Irmingersee im Juni 1955. - Ber. Dt. Wiss. Komm. Meeresforsch., 14: 255-312; Stuttgart.

Drake, C. L., Campbell, N. J., Sander, G. \& Nafe, J. E. (1963): A mid-Labrador Sea ridge. Nature, 200: 1085; London.

Fillon, R. H., Folinsbee, R. A. \& Palmer, R. (1977): Deep shelf and slope terraces off northern Labrador. - [Im Druck.]

Henderson, G. (1975): New bathymetric maps covering offshore west Greenland 59 $-69^{\circ} 30^{\prime} \mathrm{N}$. - Offshore Technology Conference Houston/Texas, Paper OTC 2223: 761 -764; Dallas.

Holtedahl, O. (1970): On the morphology of the west Greenland shelf with general remarks on the "marginal channel" problem.-Mar. Geol., 8: 155-172; Amsterdam.

Hјulströм, F. (1935): Studies on the morphological activity of rivers. - Bull. Geol. Inst. Uppsala, 25: 221-527; Uppsala.

Milliman, J. D. \& Emery, K. O. (1968): Sea levels during the past 35000 years. - Science, 162: 1121-1123; Washington.

Rvachev, V. D. (1964): Relief and bottom deposits of the shelf of southwestern Greenland. Deep Sea Res., 11: 646-653; Oxford.

Sommerhoff, G. (1975 a): Glaziale Gestaltung und marine Uberformung der Schelfbänke vor SW-Grönland. - Polarforschung, 45: 22-31; Münster.

- (1975 b): Versuch einer morphologischen Gliederung des südwestgrönländischen Kontinentalrandes. - Polarforschung, 45: 87-101; Münster.

SRivastava, S. P. (1977): Evolution of the Labrador Sea and its bearing on the early evolution of the North Atlantic. - Geophys. J. R. astr. Soc.; London. - [Im Druck.]

Tenneco Oil and Minerals Ltd. (1973): Well history report, Leif E-38. - Released to open file Oct. 1973 by Dep. Energy, Mines, Resour.; Ottawa.

Tietze, W. (1961): Uber die Erosion von unter Eis fließendem Wasser. - Mainzer Geogr. Studien, Panzer Festschr.: 125-142; Braunschweig.

VAN DER LINDEN, W. J. (1975): Crustal attenuation and sea-floor spreading in the Labrador Sea. Earth Planet. Sci. Lett., 27: 409-423; Amsterdam.

-, Fillon, R. H. \& Monahan, D. (1976): Hamilton Bank, Labrador margin: origin and evolution of a glaciated shelf. - Geological Survey of Canada paper 75-40; Ottawa. 
